\section{Revista 2020 \\ Med Vol. 28(1)}

Enero-Junio 2020
-

\title{
Evaluación de la reserva ovárica: pasado, presente y futuro*
}

\author{
Bayron Manuel Ruiz-Hoyos ${ }^{a}$
}

\begin{abstract}
Resumen: La necesidad de investigar la reserva ovárica para apoyar la consejería acerca del éxito o fracaso de los tratamientos de reproducción asistida, principalmente en pacientes con riesgo de falla reproductiva, ha generado la descripción y el uso de pruebas histológicas, basales, dinámicas y ultrasonográficas. La presente revisión tiene como objetivo describir y analizar las diferentes pruebas de evaluación de la reserva ovárica, sus limitaciones y ventajas, y ampliar la información referente a las que, por sus características y resultados, son más utilizadas en la actualidad. Para cumplirlo se realizó búsqueda en bases de datos, incluyendo investigaciones originales, revisiones sistemáticas, metaanálisis, reportes de casos y consensos de expertos, en inglés y español, desde 1980. En cuanto a los resultados, puede decirse que pruebas como la biopsia ovárica, medición de Inhibina B, test de estímulo con FSH o con GnRH, y con citrato de clomifeno, han caído en desuso; la medición de la FSH, si bien todavía utilizada, claramente tiene menor capacidad predictiva que el Conteo de Folículos Antrales (CFA) y la Hormona Antimülleriana (HAM). Se concluye que el CFA y la medición de ham son las pruebas más recomendadas, con mejor capacidad predictiva y que, no obstante algunas limitaciones, permiten un acercamiento a la estimación de la respuesta al estímulo ovulatorio. Se plantean opciones para el futuro que permitirán, a mediano plazo, resolver los interrogantes sobre la "verdadera" reserva ovárica.
\end{abstract}

Palabras clave: reserva ovárica; hormona antimülleriana; conteo de folículos antrales; estímulo ovulatorio

Fecha de recepción: 15/02/2020 Fecha de aprobación: 12/03/2020

Disponible en línea: 11/09/2020

Cómo citar: Ruiz-Hoyos BM. Evaluación de la reserva ovárica: pasado, presente y futuro. Rev. Med. [Internet]. 11 de septiembre de 2020 [citado 11 de septiembre de 2020];28(1). Disponible en: https:// revistas.unimilitar.edu.co/index.php/rmed/article/view/4605

* Artículo de revisión

a Autor de correspondencia. MD, Gineco-obstetra, magíster en Educación, profesor asociado, Universidad del Quindío. Armenia, Colombia. Correo electrónico: bruiz58@yahoo.com.mx ORCID: https://orcid.org/0000-0002-8237-4553 


\title{
Ovarian Reserve: Past, Present and Future
}

\begin{abstract}
The need to investigate the ovarian reserve to support counseling about the success or failure of assisted reproduction systems has generated the description and use of histological, basal, dynamic, and ultrasound text. The purpose of this review is to describe and analyze the different tests for evaluating ovarian reserve, their limitations and advantages, and to expand the information regarding those that, due to their characteristics and results, are most widely used today. To achieve this, databases searches were carried out, including for original research, systematic reviews, metanalyses, case reports and expert consensus, in English and Spanish, since 1980. Regarding the results, it can be said that tests such as ovarian biopsy, measurement of Inhibin B, FSH or GnRH stimulation test, and with clomiphene citrate, have fallen into disuse. FSH measurement, although still used, clearly has a lower predictive capacity than the Antral Follicle Count (AFC) and the Antimüllerian Hormone (AMH). It is concluded that the AFC and the AMH measurement are the most recommended tests, with better predictive capacity and that, despite some limitations, they allow closer estimation of the response to ovulatory stimulus. Options for the future that will allow solving the questions in the medium term about the "true" ovarian reserve are proposed.
\end{abstract}

Keywords: ovarian reserve; antimülleriana hormone; antral follicle count; ovulatory stimulus

\section{Avaliação da reserva ovariana: passado, presente e futuro}

Resumo: A necessidade de pesquisar sobre a reserva ovariana para apoiar o aconselhamento acerca do sucesso ou do fracasso dos tratamentos de reprodução assistida, principalmente em pacientes com risco de falha reprodutiva, vem gerando a descrição e o uso de exames histológicos, basais, dinâmicos e ultrassonográficos. Esta revisão tem o objetivo de descrever e analisar os diferentes exames de avaliação da reserva ovariana, suas limitações e vantagens, e ampliar a informação referente aos que, por suas características e resultados, são mais utilizados na atualidade. Para isso, foi realizada busca em base de dados, que incluiu pesquisas originais, revisões sistemáticas, meta-análise, relatos de caso e consenso de especialistas, em inglês e em espanhol, desde 1980. Quanto aos resultados, pode-se dizer que os exames como biópsia ovariana, a medição de Inibina B, exame de estímulo com FSH ou com GnRH, e com citrato de clomifeno estão em desuso; a medição da FSH, embora ainda utilizada, claramente tem menor capacidade preditiva que a contagem de folículos antrais (CFA) e o hormônio antimülleriano (HAM). Conclui-se que o CFA e a medição do HAM são os exames mais recomendados, com melhor capacidade preditiva e que, apesar de algumas limitações, permitem uma aproximação da estimativa da resposta ao estímulo da ovulação. São apresentadas opções para o futuro que possibilitarão, em médio prazo, resolver as questões sobre a "verdadeira" reserva ovariana.

Palavras-chave: reserva ovariana; hormônio antimülleriano; contagem de folículos antrais; estímulo da ovulação 


\section{Introducción}

La fertilidad femenina declina a través de los años, fenómeno atribuido en mayor parte a la reducción del número de folículos ováricos, producto de la disminución de la reserva de folículos primordiales, que afecta el desarrollo potencial de la unidad folicular y las probabilidades de lograr embarazo (1).

Antes de realizar un tratamiento de Reproducción Asistida (RA), es fundamental establecer un pronóstico del éxito del estímulo ovulatorio, tasas de fertilización e implantación, cancelación del ciclo e hiperestimulación ovárica; todo lo cual justifica investigar la Reserva Ovárica (во) para apoyar la consejería referente al pronóstico de éxito o fracaso, máxime en pacientes con riesgo de falla reproductiva (2).

Es pertinente que se instruya a la pareja en el sentido de que, si bien los biomarcadores de Ro tienen alta capacidad predictiva sobre la respuesta ovárica al estímulo ovulatorio, esta es menor en relación con la posibilidad de lograr el embarazo (1-3).

El objetivo fundamental de la presente revisión es efectuar un análisis histórico y práctico sobre el uso de las pruebas de ro y, en lo posible, caracterizar su estado actual y perspectivas para el futuro.

\section{Metodología}

Se realizó una búsqueda bibliográfica en las bases de datos Google Scholar, Science Direct, RIMA, PubMed, en idiomas español e inglés; se incluyeron investigaciones originales, revisiones sistemáticas y metaanálisis, reportes de caso y consensos de expertos, utilizando como palabras clave: "reserva ovárica", "hormona antimülleriana", "hormona folículo estimulante", "conteo de folículos antrales", "Ovarian Reserve", "Anti-Mullerian Hormone", "Follicle Stimulating Hormone", "Antral Follicle Count"; teniendo en cuenta la antigüedad del concepto objeto de la revisión de tema, se incluyeron publicaciones a partir de 1980.

\section{Resultados}

\section{¿Qué es la reserva ovárica?}

El concepto de reserva ovárica (RO) describe la dotación natural de folículos ováricos, se vincula estrechamente con la edad de la mujer y se considera el factor más importante en el resultado reproductivo (3), se relaciona directamente con el número de folículos primordiales presentes en el ovario (1) y se evidencia por la respuesta ovárica al estímulo con gonadotrofinas; de tal forma que la baja respuesta al estímulo es un indicador de la disminución de la ro (4); a su vez, el concepto de "pobre respuesta ovárica" (poor ovarian response, POR) varía ampliamente en las publicaciones $(1,5,6)$.

\section{¿Cómo se determina la reserva ovárica?}

Se puede estimar la Ro tomando como base la edad de la mujer, sus antecedentes reproductivos y la respuesta previa al estímulo ovulatorio. Las mujeres de edad reproductivamente avanzada producen menor cantidad de óvulos y los embriones obtenidos tienen menor potencial implantatorio; esta disminución de la calidad oocitaria se acompaña de una elevada incidencia de aneuploidias, monosomías y trisomías (7). Los eventos relacionados con la disminución de la RO: cirugías en ovario (resección en cuña, cistectomías, drilling), el acortamiento de los ciclos menstruales, los tratamientos oncológicos con quimio y radio terapia, predicen una baja respuesta al estímulo ovulatorio (8). El análisis de ciclos previos de estímulo para fertilizacion in vitro (FIV), aporta información importante para estimar la Ro y la futura respuesta ovulatoria $(5,8)$.

Diversas formas de identificar la Ro han sido descritas; pueden ser histológicas, basales, dinámicas y ultrasonográficas (9), algunas han caído en desuso y entre ellas están las siguientes.

\section{Biopsia Ovárica}

El conteo de folículos primordiales presentes en una muestra tomada del ovario es técnicamente impráctico, teniendo en cuenta que la distribución 
de los folículos no es homogénea en el ovario; además de las implicaciones negativas de tomar una muestra de tejido ovárico en una mujer con interés reproductivo (10), algunas publicaciones sobre la Ro, mencionan la biopsia ovárica solo para recomendar que no sea utilizada con ese fin $(1,3,10)$.

\section{Prueba de estímulo con citrato de clomifeno (Clomiphene Citrate Challange Test, CCCT)}

Consiste en la medición de la FsH el día tercero del ciclo, para luego suministrar a la paciente citrato de clomifeno $100 \mathrm{mg}$ del quinto al noveno día, y efectuar una nueva medición de FSH el día décimo del ciclo (11); se considera anormal un valor igual o superior a $26 \mathrm{mu} / \mathrm{ml}$ en la $\mathrm{FSH}$ del día décimo; sin embargo, otras interpretaciones han sido propuestas (1). En la actualidad su uso ha disminuido notoriamente (3).

\section{Prueba de estímulo con análogo de GnRH (GnRH Analog Stimulation Test, GAST)}

Se basa en la administración de acetato de leuprolide en su fase agonista, para evaluar la respuesta en los niveles de FSH, LH, estradiol y su valor pronóstico en el ciclo respectivo (12). No es recomendado ni utilizado en vista de su costo, el tiempo que conlleva y las ventajas de otras pruebas basales (3).

\section{Prueba de estímulo con FSH externa (Exogenous Follicle Stimulating Hormone Ovarian Reserve Test, EFORT).}

Consiste en administrar un bolo de 300 UI de FSH purificada y medir el incremento en la concentración de estradiol a las 24 horas respecto al valor basal (13). El tiempo y costo que implica, además de existir pocos estudios al respecto, lo hacen una alternativa poco o nada utilizada (3).

\section{FSH}

La medición de la FSH en fase folicular temprana (generalmente día tres del ciclo menstrual) ha sido una de las más utilizadas formas de evaluar la Ro; su uso se basa en que el envejecimiento ovárico se acompaña de menor producción de Inhibina в en los folículos antrales, lo que altera el feed-back para la secreción de gonadotrofinas y permite la elevación de la FSH en fase folicular temprana (14). No hay un valor absoluto de FSH que se correlacione con la falta de respuesta ovárica y el pronóstico reproductivo $(1,15)$; además, la estratificación de los valores entre 10 y 20 ui/l, arroja resultados controversiales $(15,16)$.

Se recomienda hacer la prueba entre los días 2-5 del ciclo, cuando los niveles de FsH son más altos, antes de iniciar la supresión por el estradiol (17), sin embargo, lo más frecuente es tomar la muestra el día $3^{\circ}$.

Tiene mejor pronóstico la mujer joven con FSH elevada, que la mujer mayor con FsH "normal" (16); de hecho, un valor aislado de FSH alta en una mujer $<40$ años, no predice una pobre respuesta o incapacidad para lograr el embarazo.

Actualmente, aunque su uso continúa, los estudios reportan que la FSH basal no supera al conteo de folículos antrales (AFC) y la Hormona Antimülleriana (Амн) como test de evaluación de la Ro $(1,4,6)$

\section{Estradiol}

La determinación del estradiol basal (días 2-5 del ciclo) ha sido propuesta como predictor del resultado del estímulo ovulatorio, con base en el acortamiento de la primera fase del ciclo; se ha supuesto que implica una "condensación" del reclutamiento de folículos y por ende altos niveles de estradiol en la fase folicular temprana, que incluso pueden enmascarar una elevación de la FSH (18); sin embargo, aunque algunos estudios demuestran que niveles basales de estradiol $\geq 75 \mathrm{pg} / \mathrm{m}$ se relacionan con bajas tasas de embarazo y mayor cantidad de ciclos cancelados (18), en un importante número de trabajos se encuentra poca capacidad predictiva para la baja respuesta y la falla reproductiva $(1,3)$.

\section{Inhibina $B$}

La Inhibina B es producida por las células granulosas de los folículos en desarrollo y se han encontrado sus niveles disminuidos en mujeres con baja RO (15). El valor propuesto como punto de corte de Inhibina $\mathrm{B}$, el tercer día del ciclo, es: $\geq 45 \mathrm{pg} / \mathrm{ml}$; 
valores inferiores se acompañan de menor número de oocitos recuperados, mayor tasa de cancelación de ciclos y menor tasa de embarazos (19). Aunque se han demostrado niveles más bajos de Inhibina $B$ en mujeres de edad mayor y se relacionan con la Ro disminuida (15), existe controversia sobre su utilidad, por los resultados contradictorios de los estudios $(1,20)$.

\section{Hormona Antimülleriana}

La AMH se produce en las células de la granulosa de los folículos preantrales y antrales tempranos del ovario humano y juega un importante papel en la foliculogénesis humana (21). En la mujer, la AMH actúa mediante la regulación del número de folículos que se desarrolla en una cohorte, previniendo el agotamiento del pool de folículos primordiales $(4,22)$. Hay niveles más altos en la pubertad y estos decrecen en la vida reproductiva, hasta ser indetectables en la menopausia (21).

Se ha encontrado que tiene poca variabilidad intraciclo, por lo que la medición se puede realizar en cualquier momento del ciclo (23); sus valores pueden disminuir en fumadoras y por la ingestión de alcohol (24); en obesas, aunque los valores se encuentran disminuidos, puede ser un efecto de dilución por mayor volumen sanguíneo, como sucede con otras mediciones hormonales en sangre $(23,24)$.

Dadas sus características, la AMH ha sido propuesta, entre otros usos, como indicador de ro, Síndrome de Ovario Poliquístico (sop) y predictor de hiperrespuesta ovárica (Síndrome de $\mathrm{Hi}$ perestimulación Ovárica, SHEO) (25). Los valores sugeridos como predictores de respuesta ovulatoria normal fluctúan entre 1 y $3,5 \mathrm{ng} / \mathrm{ml}(24,25)$, mientras resultados $<1 \mathrm{ng} / \mathrm{ml}$ predicen baja respuesta, más evidente con valores $<0,5 \mathrm{ng} / \mathrm{ml}$; el punto de corte para la hiperrespuesta (sHEO) es $>$ $3,5 \mathrm{ng} / \mathrm{ml}(24,25)$.

Ha existido controversia sobre el tipo de prueba diagnóstica utilizada para determinar la $\mathrm{AMH}$, lo que puede afectar el valor calculado, por lo que se recomienda establecer puntos de corte basados en los resultados de la población en cada centro de reproducción (24-26).
En la actualidad, la mayoría de los estudios confieren a la AMH la condición de mejor predictor de la respuesta ovárica (24-26).

\section{Volumen ovárico (VO)}

Existe importante disminución del vo desde los 40 años (27), la cual puede ser observada antes de las elevaciones de la FSH (10). Son diversos los puntos de corte del vo para la predicción de la POR $(1,28)$; la mayoría coincide en que un vo $<$ de $3 \mathrm{~cm} 3$ (sumatoria de ambos ovarios) se acompaña de una alta tasa de cancelación de ciclos y una baja cifra de oocitos recuperados; no obstante, la razón de probabilidades para un resultado positivo (pobre respuesta) es baja (1). En la actualidad, aunque se le confiere un papel complementario en la evaluación de la Ro (28), no es un estudio recomendado de rutina $(3,6,7,26)$.

\section{Conteo de folículos antrales (Antral Follicle Count, AFC)}

En 1998 Chang (29) introduce en la práctica clínica el AFC, en calidad de estimativo de la respuesta ovárica y del pronóstico reproductivo. El AFC tiene relación directa con la posibilidad de obtener una buena respuesta ovárica y el número de óvulos recuperados, e inversa con la edad de la mujer (27); también ha demostrado ser buen predictor de la mala respuesta ovulatoria, principalmente en mujeres de edad avanzada (27). Se acostumbra y recomienda realizar el conteo de folículos antrales en la fase folicular temprana, al inicio del estímulo ovulatorio; es innecesario practicar conteos en ciclos subsiguientes, dado que existe poca variación interciclo (30). Los autores han discrepado respecto al diámetro de los folículos que deben ser medidos $(1,27)$; sin embargo, la mayoría utiliza el conteo de los folículos de 2-10 mm (31,32). Broekmans et al sugieren la medición y conteo de los folículos de 2 a $10 \mathrm{~mm}$, en los días 2 a 4 del ciclo, informando la sumatoria de los dos ovarios, con equipos de ultrasonografía 2D y transductor de $7 \mathrm{mhtz}$, en pacientes con ciclos regulares que no presenten patología susceptible de generar crecimiento ovárico anormal (Ej: endometriomas) ni resección de ovarios (31). Coelho et al, recomiendan el conteo manual 
de los folículos de 2 a $10 \mathrm{~mm}$ con equipos 2D o 3D, y sugieren evaluar el AFC en cualquier momento del ciclo, aunque aclaran que el conteo es más fácil en fase folicular temprana, al reducir la posibilidad de observar quistes foliculares o cuerpo lúteo (32).

A diferencia del diagnóstico ecográfico de Ovario Poliquístico, en el que se han establecido nuevos puntos de corte para el conteo de folículos antrales, teniendo en cuenta los avances en la tecnología ecográfica (33), los puntos de corte del AFC no se han modificado; esto porque, aun empleando las nuevas técnicas de ultrasonografía 3D (multiplanar view, inverted mode, Virtual Organ Computer-aided Analysis, vocal, Sonography-Based Automated Volume Calculation, SonoAVC), no existen diferencias sustanciales respecto a la técnica "2D" en cuanto al número de folículos antrales identificados $(24,31,32,34)$. Actualmente, el AFC solo es superado por la AMH en su capacidad de predecir la respuesta ovárica al estímulo ovulatorio $(24,26,35)$.

\section{¿Para qué se utiliza la determinación de la reserva ovárica?}

Su uso principal, que ha originado el desarrollo de las pruebas descritas para establecer la Ro con buenos resultados, excepto algunas excepciones (36), ha sido la evaluación predictiva de la respuesta al estímulo ovulatorio en los tratamientos de RO (37); el advenimiento de la medición de AMH como el principal marcador de la Ro, ha motivado la exploración de su utilidad, sin resultados concluyentes, en la predicción de la llegada de la menopausia; coadyuvante en el diagnóstico de ovario poliquistico, o indicador de la pérdida de fertilidad a consecuencia del tratamiento en pacientes oncológicas $(25,38)$; tampoco los estudios recomiendan emplearla como prueba de evaluación del riesgo de pérdida repetida de embarazo (38).

Un aspecto en que coincide la literatura actual, es en no recomendar la medición de AMH como prueba de evaluación de la fertilidad en mujeres de edad reproductiva, no infértiles $(25,26,38)$.

\section{¿Qué hacer ante una baja Ro?}

Se describen a continuación algunos de los esfuerzos de la comunidad científica, con miras a superar el obstáculo que en la actualidad nos plantea el hallazgo de la disminución de la ro.

\section{¿Es posible mejorar el resultado reproductivo en las mujeres con baja RO?}

La mala repuesta al estímulo ovulatorio, estimada a partir de las pruebas de evaluación de la Ro, ha sido siempre un reto a superar en los tratamientos de RO; estrategias utilizadas para mejorar la respuesta ovárica, con resultados controversiales, incluyen: aumento de la dosis de gonadotrofinas, protocolos de estimulación suave (mild stimulation), microdosis de agonistas de la GnRH, protocolos de antagonistas de la GnRH (39); también adición de hormona de crecimiento y testosterona al ciclo de estímulo, sin mejorar sustancialmente los resultados (39); el acúmulo de oocitos o embriones en ciclos sucesivos (embryo banking), en pacientes con baja respuesta comprobada, adicionando o no el uso de ciclos naturales para la FIV, ha sido reportado con resultados aún no concluyentes (40). Recientemente, se ha desarrollado y popularizado la doble estimulación en un mismo ciclo, utilizando un protocolo de baja estimulación al inicio del ciclo y otro nuevo estímulo inmediatamente después de la aspiración folicular; esto con el fin de efectuar una nueva recuperación de oocitos al final del ciclo, lo que permite una mayor cantidad de oocitos recuperados, mejorando tasas de implantación y de nacidos vivos, en pacientes con previa confirmación de baja respuesta y cancelación de ciclos. Este proceso es conocido inicialmente como "Protocolo de Shangai" (41); la evaluación de esta modalidad, también denominada "Duostim" (con algunas variantes respecto a la original), arroja resultados prometedores hasta la fecha (42).

\section{¿Qué opciones considerar en la mujer con riesgo de presentar baja RO?}

Diversas situaciones de la vida de la mujer se relacionan con la disminución de la Ro: edad (7), tabaquismo, cirugías en ovario (resección en cuña, 
cistectomías, drilling), tratamientos oncológicos con quimio y radio terapia (8); se acompañan de mal pronóstico reproductivo en general y de una baja respuesta al estímulo ovulatorio en los ciclos de tratamiento de Ro en particular.

La criopreservación de embriones -opción de preservación de fertilidad existente desde hace más de treinta años-, implica la participación del varón, o el uso de semen de donante, lo que conlleva riesgos éticos y legales ante la posibilidad de separación de la pareja o el fallecimiento de la paciente (43); es por ello por lo que en la actualidad las opciones más utilizadas y recomendadas, con miras a conservar el potencial reproductivo de la mujer cuando existen circunstancias que propician la disminución de la Ro son, principalmente, la criopreservación de oocitos y de tejido ovárico. Estas estrategias se utilizan tanto en pacientes con riesgo de disminución de la reserva ovárica por su edad o historia clínica (ej.: endometriosis), como en pacientes oncológicas, fundamentalmente mujeres jóvenes con cáncer de mama o niñas y adolescentes con enfermedades hematológicas, en quienes la posibilidad de sobrevida hoy día es alta, con el consiguiente interés reproductivo $(43,44)$.

En la paciente oncológica, los mecanismos por los cuales se produce lesión al ovario por quimio y radio terapia, incluyen básicamente el daño al pool de folículos primordiales y al estroma ovárico, aunque se ha considerado también la teoría de la activación del reclutamiento folicular, con depleción de la reserva folicular (45). Entre los agentes quimioterapéuticos, los alquilantes se han relacionado en mayor medida con el daño ovárico; por su parte la radiación ionizante produce efecto tóxico a dosis de dos o más Grays (45); una medida utilizada como protección del daño gonadal en niñas sometidas a quimioterapia, ha sido la administración de análogos de la GnRH, con la intención de producir un estado de latencia de los folículos primordiales y protegerlos del daño citotóxico (44); esta estrategia ha demostrado su efecto en la protección gonadal para los tratamientos con sustancias alkilantes (46).

De acuerdo con las guías de la Asociación Americana de Oncología Clínica (Asco por sus siglas en inglés) las recomendaciones para preservación de la fertilidad en mujeres con cáncer son (47):

a. Criopreservación de embriones.

b. Criopreservación de óvulos no fertilizados. Aclaran que los protocolos de estímulo ovulatorio se pueden iniciar en cualquier momento del ciclo y que es recomendable, en los casos de cáncer de mama, utilizar protocolos de estímulo que produzcan bajos niveles de estrógenos.

c. Ooforopexia (transposición ovárica). Se ofrece cuando la irradiación pélvica es parte del tratamiento del cáncer. Debe ser realizada tan cerca del tratamiento como sea posible. Se recomienda informar a las pacientes que la técnica no siempre es exitosa.

d. Cirugía conservadora. Realizar cirugías menos radicales: Cistectomía ovárica en estadios tempranos de cáncer ovárico.

e. Supresión ovárica. La evidencia a favor de la supresión ovárica con análogos de la GnRH es controversial. En pacientes jóvenes con cáncer de mama, en quienes no sea posible utilizar métodos probados de preservación de la fertilidad, como criopreservación de oocitos, embriones o tejido ovárico, se debe ofrecer supresión con análogos de la GnRH, para disminuir el daño ocasionado al ovario por la quimioterapia.

f. Criopreservación y transplante de tejido ovárico. La criopreservación de tejido ovárico, para su futuro trasplante, no requiere estimulación ovárica y se puede realizar de inmediato; además, no requiere madurez sexual y por ello puede ser el único método asequible para las niñas prepúberes; sin embargo, amerita mayor investigación para confirmar su seguridad en paciente con leucemias.

Con respecto a la endometriosis, se ha comprobado su relación con la infertilidad, por la interacción de múltiples factores, que incluyen alteraciones ovocitarias, endometriales y embrionarias (48); la presencia del endometrioma se acompaña de disminución progresiva de la Ro por aumento de la atresia folicular; el tejido adyacente al endometrioma muestra cambios compatibles con reducción de la densidad y función folicular, 
lo cual es independiente del efecto de compresión ejercido por el endometrioma; además, se produce reducción de la calidad oocitaria $(44,49)$. Se ha demostrado también diminución de la АMH en pacientes con endometriomas en comparación con mujeres sanas o con quistes benignos de ovario (50), todo lo cual hace más complejo el enfoque de manejo de los endometriomas, dado que su tratamiento médico no es efectivo (51) y frecuentemente se impone el manejo quirúrgico.

Múltiples publicaciones confirman la disminución de la reserva ovárica como producto de las diferentes técnicas quirúrgicas utilizadas para el manejo de los endometriomas $(44,48,49,51)$. Los valores de AMH disminuyen notoriamente después de la cirugía con respecto a las mediciones previas, y si bien se ha demostrado su recuperación, no se llega a los niveles pre quirúrgicos (52); esta declinación de la Ro es más notoria en pacientes con mayor grado de severidad de la endometriosis o endometriomas bilaterales (44). Es por ello por lo que en pacientes con interés reproductivo se recomienda un enfoque conservador en la cirugía para endometriomas, que incluye posponer la cirugía hasta una fecha cercana al tratamiento de fertilidad; evitar la cirugía en pacientes asintomáticas o con sintomatología tolerable; utilizar con cautela las energías durante la cirugía (44). Además, se recomienda que la intervención sea realizada por el cirujano más experimentado (52); en cuanto a la técnica quirúrgica, hay controversia entre los métodos ablativos y la cistectomía, aunque existe mayor evidencia a favor de esta última $(44,49,52)$.

\section{Conclusiones}

Los resultados de los metaanálisis (4) y las revisiones sistemáticas $(1,24)$, así como los consensos de expertos $(3,6,7,26)$ relacionados con la Ro, coinciden en que no existe un marcador único que prediga la mala respuesta ovárica y el fracaso reproductivo, con suficiente certeza como para no aconsejar el inicio de un ciclo de FIV; máxime teniendo en cuenta casos publicados que contradicen las predicciones basados en los resultados de las pruebas de Ro $(25,26,36)$.
Limitaciones a tener en cuenta en los biomarcadores más utilizados son la falta de estandarización de las mediciones de la AMH $(24-26,37)$ y la dependencia del AFC de la experticia del operador $(25,31,32)$.

Es amplio el panorama de pruebas diseñadas para evaluar la Ro y predecir la respuesta ovárica al estímulo de ovulación para los tratamientos de Medicina Reproductiva; sin embargo, es claro que la AMH y el AFC han demostrado el mejor desempeño para estos fines $(6,24-26)$. Diversas publicaciones evidencian que la AMH cada vez toma más fuerza como el marcador "ideal" $(24,40)$. Hay consenso en que las pruebas de Ro no deben ser utilizadas en mujeres sin infertilidad como "prueba de fertilidad" $(25,26,38)$.

Se cuenta en la actualidad con alternativas para mejorar el resultado reproductivo en los tratamientos de Ro en mujeres con evidencia de disminución de la Ro $(39,40)$; también es posible minimizar los efectos de eventos de la vida reproductiva de la mujer que irremediablemente llevarán a disminuir o abolir la reserva ovárica por mecanismos físicos o farmacológicos (43-47) o quirúrgicos $(44,52)$.

\section{Retos para el futuro}

La reflexión sobre la posibilidad de fortalecer la capacidad predictiva de la pruebas de Ro, con miras a una mejor consejería acerca de los resultados de los tratamientos, cuestionando los aspectos que rodean el concepto de "reserva ovárica" (53); interrogando también las relaciones entre el verdadero pool de folículos primordiales y las pruebas que suponen dar cuenta de ellos (54), han motivado el surgimiento de marcadores relacionados con la dinámica del ciclo de estímulo $(55,56,57)$; estos, si bien ameritan al menos un ciclo previo de tratamiento para ser utilizados en la predicción de la respuesta ovulatoria de un ciclo subsiguiente, afianzan la importancia de evaluar las pruebas de ro desde dos dimensiones, interrelacionadas pero separadas: el concepto biológico basado en la noción de que el número de folículos en el ovario humano declina con el tiempo, hecho que podemos medir con pruebas de laboratorio o equipos ultrasonográficos; y el concepto clínico, el cual 
solo podemos estimar según los resultados reproductivos (58).

Una opción recientemente publicada, que, incluyendo las pruebas de reserva ovárica, estratifica las pacientes de acuerdo a sus condiciones individuales para la predicción de la baja respuesta, y permite seleccionar el protocolo de estímulo adecuado, es la del grupo Poseidon (Patient Oriented Strategies Encompassing IndividualizeD Oocyte Number) (59); este propone cuatro tipos de pacientes de bajo pronostico reproductivo, tomando como base la edad, los marcadores de reserva ovárica (AFC, $\mathrm{AMH})$, y el antecedente de respuesta "subóptima" previa a un protocolo de estimulación standard (60).

Queda aún por definir el papel de algunas intervenciones o productos farmacológicos en la restauración de la Ro. Publicaciones acerca del uso de Plasma Rico en Plaquetas de origen autólogo (61), acupuntura (62) y hierbas medicinales chinas (63), informan resultados prometedores, con resultados que muestran mejoría en los marcadores de reserva ovárica, con mayores de tasas de oocitos recuperados, y embarazos clínicos; sin embargo, aún no existe suficiente evidencia para recomendar su uso. Por otra parte, no obstante el entusiasmo que algunas publicaciones despiertan acerca de los efectos de la Dehidroepiandrosterona (DHEA) para revertir la disminución de la Ro (64), los informes de los metaanálisis y revisiones sistemáticas, aún son controversiales (65-67). Estas y otras intervenciones y productos farmacéuticos o naturales, podrían impactar directa o indirectamente en la dinámica de la reserva folicular a través de rutas genéticas, cuyo conocimiento avanza con rapidez en la actualidad $(53,54)$.

\section{Referencias}

[1] Broekmans F, Kwee J, Hendriks D, Mol B, Lambalk C. A systematic review of test predicting ovarían reserve and IVF autcome [Internet]. Human Reproduction Update. 2006;12(6):685-718. Dor: https://doi.org/10.1093/ humupd/dml034

[2] McIlveen M, Skull J, Ledger W. Evaluation of the utility of multiple endocrine and ultrasound measures of ovarian reserve in the prediction of cycle cancellation in a high-risk IVF population [Internet]. Human
Reproduction. 2007;22(3):778-785. DoI: https:/doi. org/10.1093/humrep/del435

[3] Sills E, Alper M, Walsh A. Ovarian reserve screening in infertility: Practical applications and theoretical directions for research [Internet]. European Journal of Obstetrics \& Gynecology and Reproductive Biology. 2009;146:30-36. DoI: https://doi.org/10.1016/j.ejogrb.2009.05.008

[4] Verhagen T, Hendriks D, Bancsi L, Mol B, Broekmans F. The accuracy of multivariate models predicting ovarian reserve and pregnancy after in vitro fertilization: a meta-analysis [Internet]. Human Reproduction Update. 2008;14(2):95-100. DoI: https://doi.org/10.1093/ humupd/dmn001

[5] Ferraretti A, La Marca A, Frauser B, Tarlatzis B, Nargund $\mathrm{G}$, Gianaroli L, on the belhalf of the ESHRE working group on Poor Ovarian Response Definition. ESHRE consensus on the definition of "poor response" to ovarian stimulation for in vitro fertilization: the Bologne criteria [Internet]. Human Reproduction. 2011; 26(7):1616-24. DOI: https://doi.org/10.1093/humrep/ der092

[6] La Marca A, Ferraretti A, Palermo R, Ubaldi F. The use of ovarian reserve markers in IVF clinical practice: a national consensus [Internet]. Gynecol Endocrinol. 2016;32(1):1-5. DoI: https://doi.org/10.3109/09513590.2 015.1102879

[7] Podfigurna A, Lukaszukb K, Czyzyka A, Kunickic M, Maciejewska-Jeskea M, Jakielc G, et al. Testing ovarian reserve in pre-menopausal women: why, whom and how? [Internet]. Maturitas. 2018; 109:112-17. DOI: https://doi.org/10.1016/j.maturitas.2017.11.014

[8] Younis J, Ben-Ami M, Ben-shlomo I. The Bologna criteria for poor ovarian response: a contemporary critical appraisal [Internet]. Journal of Ovarian Research. 2015;8(76):2-10. DOI: https://doi.org/10.1186/s13048015-0204-9

[9] Johnson N, Bagrie E, Coomarasamy A, Bhattacharya S, Shelling A, Jessop S, et al. Ovarian reserve tests for predicting fertility outcomes for Assisted Reproductive Technology: the International Systematic Collaboration of Ovarian Reserve Evaluation protocol for a systematic review of test accuracy [Internet]. BJoG. 2006; 113:1472-80. DoI: https://doi.org/10.1111/j.14710528.2006.01068.x

[10]Lass, A. Assesment of ovarian reserve - is there a role for ovarian biopsy? [Internet]. Human Reproduction. 2001;16(6):1055-57. DOI: https://doi.org/10.1093/ humrep/16.6.1055

[11] Navot D, Rosenwaks Z, Margalioth E. Prognostic assessment of female fecundity [Internet]. Lancet. 
1987;330(8560):645-47. DOI: https://doi.org/10.1016/ s0140-6736(87)92439-1

[12] Padilla S, Bayati J, Garcia J. Prognostic value of the early serum estradiol response to leuprolide acetate in in vitro fertilization [Internet]. Fertility and Sterility. 1990;53(2): 288-94. DoI: https://doi.org/10.1016/s00150282(16)53283-X

[13]Fanchin R, Ziegler D, Olivennes S, Taieb J, Dzik A, Frydman R. Exogenous follicle stimulating hormone ovarian reserve test (EFORT): a simple and reliable screening test for detecting 'poor responders' in in-vitro fertilization [Internet]. Hum Reprod. 1994;9(9):1607-11. DoI: https://doi.org/10.1093/oxfordjournals.humrep.a138760

[14] Koning C, Pop-Snijders C, Schoemaker J, Lambalk C. Elevated FSH concentrations in imminent ovarian failure are associated with higher $\mathrm{FSH}$ and $\mathrm{LH}$ pulse amplitude and response to GnRH [Internet]. Human Reproduction. 2000;15(7): 1452-56. DoI: https://doi. org/10.1093/humrep/15.7.1452

[15]Bukman A, Heineman M. Ovarian reserve testing and the use of prognostic models in patients with subfertility [Internet]. Human Reproduction Update. 2001;7(6):581-90. DOI: https://doi.org/10.1093/humu$\mathrm{pd} / 7.6 .581$

[16]Abdalla H, Thum M. An elevated basal FSH reflects a quantitative rather than qualitative decline of the ovarian reserve [Internet]. Human Reproduction. 2004;19(4):893-98. DOI: https://doi.org/10.1093/ humrep/deh141

[17] Magee B, Reid R. A Pragmatic Approach to Hormonal Testimg in the Assessment of Disorders of Female Reproduction [Internet]. Int J Womens Health Wellness. 2016;2(3):022. DoI: https://doi.org/10.23937/2474$1353 / 1510022$

[18] Licciardi F, Lui H, Rosenwaks Z. Day 3 estradiol serum concentrations as prognosticators of ovarian stimulation response and pregnancy outcome in patients undergoing in vitro fertilization [Internet]. Fertility and Sterility. 1995;64(5):991-94. DOI:10.1016/s0015https://doi.org/0282(16)57916-3

[19] Corson S, Gutmann J, Batzer F, Wallace H, Klein N, Soules M. Inhibin B as a test of ovarian reserve for infertile women [Internet]. Human Reproduction. 1999;14(11):2818-21. DOI: https://doi.org/10.1093/ humrep/14.11.2818

[20]Al-Azemi M, Killick S, Duffy S, Pye C, Refaat B, Hill N. Multi-marker assessment of ovarian reserve predicts oocyte yield after ovulation induction [Internet]. Human Reproduction. 2011;26(2):414-22. DoI: https:// doi.org/10.1093/humrep/deq339
[21]Fouany M, Sharara F. The role of anti-Mullerian hormone in assisted reproductive medicine [Internet]. Middle East Fertility Society Journal. 2011;16:103-106. DOI: https://doi.org/10.1097/GCO.0b013e3283384911

[22]Ducatelli M, Marchetti I, Benecia H, Gismondi F, Neuspiller N, Coco R. La influencia de Амн en los resultados de FIV/ICSI [Internet]. Reproducción. 2009;24: 182 - 84. Disponible en: http://www.fecunditas.com. ar/publicados/influencia_de_amh.pdf

[23]Lambert-Messerlian G, Plante B, Eklund E, Raker C, Moore R. Levels of antimüllerian hormone in serum during the normal menstrual cycle [Internet]. Fertil Steril. 2016;105:208-13. DoI: https://doi.org/10.1016/j. fertnstert.2015.09.033

[24]Iliodromiti S, Anderson R, Nelson S. Technical and performance characteristics of anti-Müllerian hormone and antral follicle count as biomarkers of ovarian response [Internet]. Human Reproduction Update. 2015; 21(6)698-710. DoI: https://doi.org/10.1016/j.fertnstert.2014.12.114

[25]Tal R, Seifer D. Ovarian reserve testing: a user's guide. Expert Reviews [Internet]. American journal of obstetrics and Gynecology. 2017;17(2):129-140. DoI: https:// doi.org./10.1016/j.ajog.2017.02.027

[26]Practice Committee of the American Society for Reproductive Medicine. Testing and interpreting measures of ovarian reserve: a committee opinión. [Internet]. Fertility and Sterility. 2015;103(3):e9-e17. Dor: http:// dx.doi.org/10.1016/j.fertnstert.2014.12.093

[27]Kwee J, Elting M, Schats R, McDonnell J, Lambalk C. Ovarian volume and antral follicle count for the prediction of low and hyper responders with in vitro fertilization [Internet]. Reproductive Biology and Endocrinology. 2007;5(9):1-10. DoI: https://doi. org/10.1186/1477-7827-5-9

[28]Agarwal A, Agarwal S, Verma A, Shukla RC. Total Basal Ovarian Volume as a Marker of Ovarian Reserve: Myth or Reality [Internet]. Journal of Clinical and Diagnostic Research. 2018;12(2):TC13-TC16. DOI: https://doi.org/10.7860/JCDR/2018/31482.11211

[29]Chang M-Y, Chiang C-H, Hsei T-T, Soong Y-k, Hsu $\mathrm{K}-\mathrm{H}$. Use of the antral follicle count to predict the outcome of assisted reproductive technologies [Internet]. Fertility and Sterility. 1998;69(3):576-602. DoI: https://doi.org/10.1016/s0015-0282(97)00557-8

[30]Van Disseldorp J, Lambalk C, Kwee J, Looman C, Eijkemans M, Fauser B, et al. Comparison of inter and intra-cycle variability of anti-Mullerian hormone and antral follicle count [Internet]. Human Reproduction. 2010;25(1):221-27. DoI: https://doi.org/10.1093/ humrep/dep366 
[31]Broekmans F, Ziegler D, Howles C, Gougeon A, Trew $\mathrm{G}$, Olivennes F. The antral follicle count: practical recommendations for better standardization [Internet]. Fertility and Sterility. 2010;94(3):1044-51. DOI: https://doi.org/10.1016/j.fertnstert.2009.04.040

[32]Coelho Neto M, Ludwin A, Borrell A, Benacerraf B, Dawailly D, Da Silva Costa F, et al. Counting ovarian antral follicles by ultrasound: a practical guide [Internet]. Ultrasound Obstet Gynecol. 2018;51:10-20. DOI: https://doi.org/10.1002/uog.18945

[33]Teede HJ, Misso ML, Costello MF, Dokras A, Laven J, Moran L, et al. International Pcos Network. Recommendations from the international evidence-based guideline for the assessment and management of polycystic ovary síndrome [Internet]. Fertility and Sterility. 2018;110(3):364-379. DoI: https://doi.org/10.1016/j. fertnstert.2018.05.004

[34]Bonilla-Musoles F, Castillo JC, Caballero O, Raga F, Bonilla F Jr, Dolz M, et al. (2011.). El cálculo de la reserva ovárica mediante la determinación del recuento de foliculos antrales (rfa) y la hormona anti-mülleriana (ham). Cuadernos de Medicina Reproductiva. Ecografía en Reproducción en el siglo xxi. 2011;17(1):23-37.

[35]Arce M, Loayza E, Marroquin P, Carvallo F. Conteo de folículos antrales como predictor de respuesta ovárica y de resultados de FIV/ICSI [Internet]. Revista Peruana de Ginecología y Obstetricia. 2009;55(1):37-42. DOI: https://doi.org/10.31403/rpgo.v55i266

[36]Burks H, Baker M, Quaas A, Bendikson K, Chung $\mathrm{K}$, Paulson R. The dilemma of counseling patients about poor prognosis: live birth after IVF with autologous oocytes in a 43-year-old woman with FsH levels above $30 \mathrm{miu} / \mathrm{ml}$ [Internet]. J Assist Reprod Genet. 2017;34:1185-88. DoI: https://doi.org/10.1007/s10815017-0986-3

[37]Jacobs M, Reuter L, Baker V, Craig L, Sakkas D, Surrey $\mathrm{E}$, et al. A multicentre evaluation of the Elecsys@anti-Müllerian hormone immunoassay for prediction of antral follicle count [Internet]. rbmo. 2019;38(5):84552. Dor: https://doi.org/10.1016/j.rbmo.2018.12.041

[38]Committee on Gynecologic Practice. The Use of Antimüllerian Hormone in Women Not Seeking Fertility Care. ACOG COMmittee opinion 773 [Internet]. Obstet Gynecol. 2019;133(4):e274-78. DoI: https://doi. org/10.1097/AOG.0000000000003162

[39] Özkan ZS. Ovarian stimulation modalities in poor responders [Internet]. Turkish Journal of Medical Sciences. 2019;49(4):959-962. DoI: https://doi.org/10.3906/ sag-1905-179

[40]Sadeghi MR, Oocytes/Embryos Banking: A Vague Hope for Poor Responder Women [Internet]. Journal of reproduction \& infertility. 2018;19(3):123-124. https:// www.researchgate.net/publication/327369735_OocytesEmbryos_Banking_A_Vague_Hope_for_Poor_ Responder_Women

[41]Kuang Y, Chen Q, Hong Q, Lyu Q, Ai A, Fu Y, et al. Double stimulation during follicular and luteal phases of poor responders in IVF/ICSI programmes (Shangai protocol) [Internet]. Reproducttive Biomedicine Inline. 2014;29(6):684-91. Dor: http://dx.doi.org/10.1016/j. rbmo.2014.08.009.

[42]Sfakianoudis K, Pantos K, Grigoriadis S, Rapani A, Maziotis M, Tsioulou $\mathrm{P}$, et al. What is the true place of a double stimulation and double oocyte retrieval in the same cycle for patients diagnosed with poor ovarian reserve? A systematic review including a meta-analytical approach [Internet]. Journal of Assisted Reproduction and Genetics. 2020; 37:181-204. DoI: https://doi. org/10.1007/s10815-019-01638-z

[43]Donnez J, Dolmans MM. Fertility Preservation in Women [Internet]. N Engl J Med. 2017;377(17):1657-65. DoI: https://doi.org/10.1056/NEJMra1614676

[44]Landsberg D, Fernando S, Cohen J, Rombauts L. The Role of Fertility Preservation in Women with Endometriosis: A Systematic Review [Internet]. Journal of Minimally Invasive Gynecology. 2020;27(2):362-72. DOI: https://doi.org/10.1016/j.jmig.2019.09.780

[45]Jirge PR. Role of ovarian reserve testing in cáncer survivors [Internet]. Onco Fertil J. 2019;2:3-8. DoI: https://doi.org/10.4103/tofj.tofj_1_19

[46]Sofiyeva N, Siepmann T, Barlinn K, Seli E, Ata B. Gonadotropin - Releasing Hormone Analogs for Gonadal Protection During Gonadotoxic Chemotherapy: A Systematic Review and Meta-Analysis [Internet]. Reproductive Siciences. 2019;26(7):939-53. DOI: https://doi.org/10.1177/1933719118799203

[47]Oktay K, Harvey BE, Partridge AH, Quinn GP, Reinecke J, Taylor HS, et al. Fertility Preservation in PatientsWith Cancer: Asco Clinical Practice Guideline Update [Internet]. J Clin Oncol. 2018;36(19):1994-2001 DOI: https://doi.org/10.1200/JCO.2018.78.1914.

[48]Gilabert-Estellés J. Nuevas perspectivas en la endometriosis: Desde la fisiopatología al tratamiento médico de la enfermedad [Internet]. Rev Iberoam Fert Rep Hum. 2011;28(1):15-31. Disponible en: http:// www.revistafertilidad.org/articulo/Nuevas-perspectivas-en-la-endometriosis-Desde-la-fisiopatologiacutea-al-tratamiento-meacutedico-de-la-enfermedad/29

[49]Kitajima M, Khan KN, Harada A, Taniguchi K, Inoue $\mathrm{T}$, Kaneuchi $\mathrm{M}$, et al. Association between ovarian endometrioma and ovarian reserve [Internet]. Front Biosci (Elite Ed). 2018;10:92-102. DoI: https://doi.org/ https://doi.org/10.2741/e810 
[50]Muzzi L, Di Tucci CH, Di Feliciantonio M, Galati G, Di Donato V, Musella A, et al. Antimûllerian hormone is reduced in the presence of ovarian endometriomas: a systematic review and meta-analysis [Internet]. Fertility and Sterility. 2018;110(5):932-940e1. DoI: https://doi.org/10.1016/j.fertnstert.2018.06.025

[51] Saavedra J. Controversias en el manejo Quirúrgico del endometrioma ovárico [Internet]. Rev Col Obstet Ginecol. 2002;53(4):355-66. Dor: https://doi.org/10.18597/ rcog. 635

[52]Sônmezer M, Taskin S. Fertility preservation in women with ovarian endometriosis [Internet]. Women's Health. 2015;11(5):625-631. DoI: https://doi. org/10.2217/whe.15.49

[53]Findlay J, Hutt K, Hickey M, Anderson R. What is the "ovarian reserve"? [Internet]. Fertility and Sterility. 2015;103(3):628-30. DOI: https://doi.org/10.1016/j.fertnstert.2014.10.037

[54]Monniaux D, Clément F, Dalbiés-tran R, Estienne A, Fabre S, Mansanet C, et al. The Ovarian Reserve of Primordial Follicles and the Dynamic Reserve of Antral Growing Follicles: What Is the Link? [Internet]. Biology of Reproduction. 2014;90(4):85,1-11. DOI: https://doi.org/10.1095/biolreprod.113.117077

[55]Genro VK, Grynberg M, Scheffer JB, Roux I, Frydman R, Fanchin R. Serum anti-Müllerian hormone leves are negatively related to Follicular Output RaTe (FORT) in normo-cycling women undergoing controlled ovarian hyperstimulation [Internet]. Human Reproduction. 2011;26(3):671-77. doi: https://Dor.org/10.3389/ fendo.2019.00246

[56]Biasoni V, Patriarca A, Dalmasso P, Bertagna A, Manieri $\mathrm{Ch}$, Benedetto $\mathrm{Ch}$, et al. Ovarian sensitivity index in strongly related to circulating AMH and may be used to predict ovarian response to exogenous gonadotropins in IVF [Internet]. Reproductive Biology and Endocrinology. 2011;9:112. DoI: https://doi. org/10.1186/1477-7827-9-112

[57]Alviggi C, Conforti A, Esteves S, Vallone R, Venturella R, Staiano S, et al. Understanding Ovarian Hypo-Response to Exogenous Gonadotropin in Ovarian Stimulation and Its New Proposed Marker-The Follicle-To-Oocite (foi) Index [Internet]. Front Endocrinol. 2018;9:589. DoI: https://doi.org/10.3389/fendo. 2018.00589

[58]Ulrich N, Marsh E. Ovarian Reserve Testing: A Review of the Options, Their Applications, and Their Limitations [Internet]. Clinical Obstetrics and Ginecology. 2019;62(2):228-37. DoI: https://doi.org/10.1097/ GRF.0000000000000445
[59]Alviggi C, Andersen C, Buehler K, Conforti A, De Placido $\mathrm{G}$, Esteves $\mathrm{S}$, et al. A new more detailed stratification of low responders to ovarian stimulation: from poor ovarian response to a low prognosis concept [Internet]. Fertility and Sterility. 2016;105(6):1452-53. DoI: https://doi.org/10.1016/j.fertnstert.2016.02.005

[60]Esteves S, Roque M, Bedoschi G, Conforti A, Humaidan P, Alviggi C. Defining Low Prognosis Patients Undergoing Assisted Reproductive Tecnology: PoseIdon Criteria- The Why [Internet]. Front Endocrinol. 2018;9:461. DoI: https://doi.org/10.3389/fendo.2018.00461

[61]Sfakianoudis K, Simopoulou M, Nitsos N, Rapani A, Pantou A, Vaxevanoglou T, et al. A Case Series on Platelet-Rich Plasma Revolutionary Management of Poor Responder Patients [Internet]. Gynecol Obstet Invest. 2019;84(1):99-106. DOI: https://doi.org/10.1159/000491697

[62]Jang S, Kim KH, Jun JH, You S. Acupuncture for in vitro fertilization in women with poor ovarian response: a systematic review [Internet]. Integrative Medicine Research. (2020);9(2). DoI: https://doi.org/10.1016/j. imr.2020.02.003

[63]Lu D, Zhang SH, Song DR. TMR [Internet]. Integrative Medicine. 2019;(3):e19019. DoI: https://doi. org/10.12032/TMRIM201903019

[64] Ozatik FY, Ozatik O, Yigitaslan S, Kaygisiz B, Erol K. Do Resveratrol and Dehydroepiandrosterone Increase Diminished Ovarian Reserve? [Internet]. Eurasian J Med. 2020;52(1):6-11. DoI: https://doi.org/10.5152/eurasianjmed.2019.19044

[65]Lin J, Dang Y, Guo G, Wang Z. The influence of Dehydroepiandrosterone (DHEA) supplementation for in vitro fertilization in women with diminished ovarian reserve: a meta-analysis of randomized controlled trials [Internet]. Int J Clin Exp Med. 2017;10(12):1587815885. Disponible en: http://ijcem.com/files/ijcem0059644.pdf

[66]Schwarze JA, Canales J, Crosby J, Ortega-Hrepich C, Villa S, Pommer R. dhea use to improve likelihood of IVF/ICSI success in patients with diminished ovarian reserve: A systematic review and meta-analysis [Internet]. JBRA Assisted Reproduction. 2018;22(4):369-374. DoI: https://doi.org/10.5935/1518-0557.20180046

[67]Xu L, Hu C, Liu Q, Li Y. The Effect of Dehydroepiandrosterone (DHEA) Supplementation on IVF or ICSI: A Meta-Analysis of Randomized Controlled Trials [Internet]. Geburtshilfe und Frauenheilkunde. 2019;79(7):705-712. DOI: https:/doi. org/10.1055/a-0882-3791. 